\title{
Closed essential surfaces in hyperbolizable acylindrical 3-manifolds
}

\author{
James W. Anderson \\ Faculty of Mathematical Studies, University of Southampton, \\ Highfield, Southampton SO17 1BJ, England
}

\begin{abstract}
We show that a compact hyperbolizable acylindrical 3-manifold with non-empty incompressible boundary, in which every boundary component has genus at least two, necessarily contains a closed immersed essential surface.
\end{abstract}

\section{Introduction}

The purpose of this note is to demonstrate the existence of closed immersed essential surfaces in a certain class of hyperbolizable 3 -manifolds. We state here our main result.

Theorem 4.2: $\quad$ Let $M$ be a compact hyperbolizable acylindrical 3-manifold with non-empty incompressible boundary, in which every boundary component has genus at least two. Then, $M$ contains a closed immersed essential surface.

The proof uses techniques from both the theory of Kleinian groups and the theory of 3-manifolds. The connections between these two theories were first noticed by Poincarè, were first exploited by Marden [12], and were used to great effect by Thurston in his study of the geometrization of 3manifolds. We begin in Section 2 by giving a general description of those connections which are relevant to this work.

In Section 3, we discuss the particular case of Klein-Maskit combination theory which plays a central role in the proof of Theorem 4.2, and set the discussion of Klein-Maskit combination theory in the language of the Bass-Serre theory of fundamental groups of graphs of groups. The proof of Theorem 4.2 then follows in Section 4.

We note here that Cooper, Long, and Reid [7], using different techniques, have recently established a stronger result, showing that a compact hyperbolizable 3-manifold $M$ with non-empty incompressible boundary either is covered by an I-bundle or contains a closed immersed essential surface, which then lifts to an embedded non-separating surface in a finite cover of $M$.

Acknowledgements: I would like to thank Alan Reid, Graham Niblo, and Steve Kerckhoff for helpful conversations at various points in the life of this work, and the referee for his comments. This work was partially supported by NSF grant DMS-8505550 at MSRI, and by an NSF-NATO postdoctoral fellowship at the University of Warwick. 


\section{Language}

The purpose of this section is to present some of the background material used in this work, and to take an opportunity to describe the connections between the languages of Kleinian groups and of 3-manifolds which arise throughout this paper. Many of the results described in this Section are either implicit or explicit in the literature of the subject. We begin by describing some Kleinian group basics, using Maskit [15] as our general reference, and some 3-manifold basics, using Hempel [10] and Jaco [11] as general references. We also state some basic Lemmas which allow us to easily translate from one language to the other. As is common practice, we suppress the explicit choice of a basepoint when referring to fundamental groups, assuming that some convenient choice of basepoint has been made.

\subsection{Kleinian group basics}

A Kleinian group is a discrete subgroup of $\mathbf{P} \mathbf{S L}_{2}(\mathbf{C})$, which we view as acting both on hyperbolic 3 -space $\mathbf{H}^{3}$ via orientation-preserving isometries and on the Riemann sphere $\overline{\mathbf{C}}$ via Möbius transformations. Unless explicitly noted otherwise, we assume that Kleinian groups are torsion-free; the extension of Theorem 4.2 to Kleinian groups with torsion is given in Remark 5.1.

The action of a Kleinian group $\Gamma$ partitions $\overline{\mathbf{C}}$ into the domain of discontinuity $\Omega(\Gamma)$, which is the largest open subset of $\overline{\mathbf{C}}$ on which $\Gamma$ acts discontinuously, and the limit set $\Lambda(\Gamma)$; when $\Gamma$ is infinite, $\Lambda(\Gamma)$ is the smallest non-empty closed subset of $\overline{\mathbf{C}}$ invariant under the action of $\Gamma$.

If the domain of discontinuity $\Omega(\Gamma)$ of a Kleinian group $\Gamma$ is non-empty, it contains either one, two, or countably infinitely many connected components. Two components $\Delta_{1}$ and $\Delta_{2}$ of $\Omega(\Gamma)$ are inequivalent if there does not exist $\gamma \in \Gamma$ with $\gamma\left(\Delta_{1}\right)=\Delta_{2}$.

The limit set $\Lambda(\Gamma)$ of $\Gamma$ either is finite, in which case $\Gamma$ is elementary, or is perfect and uncountable, in which case $\Gamma$ is non-elementary. A torsion-free Kleinian group is elementary if and only if it is abelian.

The following Lemma is an immediate consequence of the Ahlfors finiteness theorem [2].

Lemma 2.1 Let $\Gamma$ be a finitely generated Kleinian group with non-empty domain of discontinuity, and let $\Phi$ be a finitely generated subgroup of $\Gamma$. Then, $\Phi$ has finite index in $\Gamma$ if and only if $\Lambda(\Phi)=\Lambda(\Gamma)$.

Given a subset $X$ of $\mathbf{H}^{3} \cup \overline{\mathbf{C}}$ and a Kleinian group $\Gamma$, the stabilizer of $X$ in $\Gamma$ is the subgroup $\operatorname{st}_{\Gamma}(X)=\{\gamma \in \Gamma \mid \gamma(X)=X\}$ of $\Gamma$. We usually refer to the stabilizer of a component of $\Omega(\Gamma)$ as a component subgroup. Say that $X$ is precisely invariant under $\Phi$ of $\Gamma$ if $\operatorname{st}_{\Gamma}(X)=\Phi$ and if $X \cap \gamma(X)$ is empty for all $\gamma \in \Gamma-\Phi$. By way of example, a component of the domain of discontinuity of $\Gamma$ is always precisely invariant under its stabilizer in $\Gamma$.

Given a Jordan curve $c$ and a closed set $Z$ in $\overline{\mathbf{C}}$, say that $c$ separates $Z$ if both of the components of $\overline{\mathbf{C}}-c$ contain points of $Z$. More generally, given a Jordan curve $c$ and a pair $W$ and $Z$ of closed 
sets in $\overline{\mathbf{C}}$, say that $c$ segregates $W$ from $Z$ if $c$ does not separate either $W$ or $Z$, and if $W$ and $Z$ lie in the different closed discs in $\overline{\mathbf{C}}$ determined by $c$.

The convex hull $\mathrm{CH}_{\Gamma}$ of $\Gamma$ is the smallest non-empty convex subset of $\mathbf{H}^{3}$ which is invariant under the action of $\Gamma$. The convex core of $\Gamma$ is the submanifold $\mathrm{C}_{\Gamma}=\mathrm{CH}_{\Gamma} / \Gamma$ of $\mathbf{H}^{3} / \Gamma$, and is the smallest convex submanifold of $\mathbf{H}^{3} / \Gamma$ whose inclusion is a homotopy equivalence. Say that $\Gamma$ is convex co-compact if $\mathrm{C}_{\Gamma}$ is compact. We note that convex co-compact Kleinian groups are necessarily finitely generated and cannot contain parabolic elements.

We make note of the following useful property of convex co-compact Kleinian groups, due to Thurston; if $\Gamma$ is a convex co-compact Kleinian group with non-empty domain of discontinuity $\Omega(\Gamma)$ and if $\Phi$ is a finitely generated subgroup of $\Gamma$, then $\Phi$ is necessarily convex co-compact. A proof of this can be found in Section 7 of Morgan [20].

\subsection{Quasifuchsian groups}

A very important class of Kleinian groups are the quasifuchsian groups. A quasifuchsian group is a finitely generated Kleinian group whose limit set is a Jordan curve and which contains no element interchanging the two components of its domain of discontinuity. It is known that a quasifuchsian group without parabolic elements is necessarily convex co-compact (see for example Lemma 3.3 of Marden [12]); moreover, if $\Phi$ is a convex co-compact quasifuchsian group and if $\Delta$ is a component of $\Omega(\Phi)$, then $\Delta / \Phi$ is a closed orientable surface of genus at least two.

The following Lemma gives a useful characterization of quasifuchsian groups.

Lemma 2.2 Let $\Phi$ be a convex co-compact Kleinian group. Then, $\Phi$ is quasifuchsian if and only if it is isomorphic to the fundamental group of a closed orientable surface of genus at least two.

Proof Suppose that $\Phi$ is isomorphic to the fundamental group of a closed orientable surface of genus at least two. We begin by noting, since $\Phi$ is convex co-compact, that $\mathbf{H}^{3} / \Phi$ is closed if and only if $\Omega(\Phi)$ is empty. Combining this with the fact that the fundamental group of a closed 3 -manifold cannot be isomorphic to the fundamental group of a closed surface of positive genus (see for example Theorem 10.6 of Hempel [10]), we see $\Omega(\Phi)$ is non-empty. Lemma 1.2 of Anderson [5] gives that $\Lambda(\Phi)$ is connected, and that either $\Phi$ is quasifuchsian or $\Omega(\Phi)$ is connected. In the later case, $\Phi$ cannot be convex co-compact (see for example Greenberg [9]), and so $\Phi$ must be quasifuchsian. The converse is immediate from the definition of quasifuchsian.

Let $\Phi$ be a quasifuchsian group and let $\Theta$ be a finitely generated infinite index subgroup of $\Phi$. By Lemma 2.1, $\Lambda(\Theta)$ is a proper closed subset of $\Lambda(\Phi)$, and so each component of $\Lambda(\Phi)-\Lambda(\Theta)$ is an open arc in the Jordan curve $\Lambda(\Phi)$. Let $a$ be such an arc. Since $\Theta$ is finitely generated, there exists a non-trivial primitive element $\theta_{a}$ of $\Theta$ stabilizing $a$, that is, so that $\theta_{a}(a)=a$; in fact, $a$ is precisely invariant under $\left\langle\theta_{a}\right\rangle$ in $\Theta$. This follows from the discussion in Section V.G of Maskit [15], particularly Theorem V.G.14. We refer to $\theta_{a}$ as a boundary loxodromic of $\Theta$ in $\Phi$. We note that $\theta_{a}$ is necessarily primitive in $\Phi$. 
Using the classical uniformization theorem of Koebe for simply connected Riemann surfaces (see for example Abikoff [1]), for each component $\Delta$ of $\Omega(\Phi)$ there exists a conformal homeomorphism $f: \Delta \rightarrow U$, where $U$ is the unit disc in $\mathbf{C}$ equipped with the Poincaré metric. Each non-trivial element $f \cdot \varphi \cdot f^{-1}$ of $f \cdot \Phi \cdot f^{-1}$ keeps invariant a unique hyperbolic line $l$ in $U$, and so $\varphi$ keeps invariant a canonical arc $l_{\varphi}=f^{-1}(l)$ in $\Delta$, which is a line in the induced hyperbolic metric on $\Delta$. We refer to $l_{\varphi}$ as the axis of $\varphi$ in $\Delta$.

\subsection{Component subgroups}

Let $\Gamma$ be a convex co-compact Kleinian group, let $\Delta$ be a component of $\Omega(\Gamma)$, and let $\Phi$ be the stabilizer of $\Delta$ in $\Gamma$. Lemma 2 of Ahlfors [3] implies that $\Phi$ is finitely generated, hence convex co-compact, and that $\partial \Delta=\Lambda(\Phi)$. If in addition $\Gamma$ has connected limit set, then $\Delta$ is necessarily simply connected, as it is a component of the complement of a closed connected subset of $\overline{\mathbf{C}}$, and so by Lemma $2.2 \Phi$ is quasifuchsian. Conversely, suppose that each component subgroup of $\Gamma$ is quasifuchsian. The boundary of each component of $\Omega(\Gamma)$ is then connected, hence each component of $\Omega(\Gamma)$ is simply connected, and so $\Lambda(\Gamma)$ is connected. We summarize this discussion in the following Lemma.

Lemma 2.3 Let $\Gamma$ be a convex co-compact Kleinian group with non-empty domain of discontinuity. Then, a component of $\Omega(\Gamma)$ is simply connected if and only if its stabilizer is quasifuchsian. Moreover, each component subgroup of $\Gamma$ is quasifuchsian if and only if $\Lambda(\Gamma)$ is connected, or equivalently, each component subgroup of $\Gamma$ is quasifuchsian if and only if each component of $\Omega(\Gamma)$ is simply connected.

It follows from Theorem 3 of Maskit [14] that if $\Phi_{1}$ and $\Phi_{2}$ are component subgroups of a convex co-compact Kleinian group $\Gamma$, stabilizing components $\Delta_{1}$ and $\Delta_{2}$ of $\Omega(\Gamma)$, then $\partial \Delta_{1} \cap \partial \Delta_{2}=$ $\Lambda\left(\Phi_{1}\right) \cap \Lambda\left(\Phi_{2}\right)=\Lambda\left(\Phi_{1} \cap \Phi_{2}\right)$, and so $\Lambda\left(\Phi_{1}\right) \cap \Lambda\left(\Phi_{2}\right)$ is empty if and only if $\Phi_{1} \cap \Phi_{2}$ is trivial, which holds if and only if $\partial \Delta_{1} \cap \partial \Delta_{2}$ is empty.

Let $\theta$ be a boundary loxodromic of $\Phi_{1} \cap \Phi_{2}$; note that $\theta$ is a boundary loxodromic of $\Phi_{1} \cap \Phi_{2}$ in $\Phi_{1}$ if and only if it is a boundary loxodromic of $\Phi_{1} \cap \Phi_{2}$ in $\Phi_{2}$. Let $A_{\theta}$ be the Jordan curve formed by taking the union of the axes of $\theta$ in $\Delta_{1}$ and $\Delta_{2}$, together with the fixed points of $\theta$. We refer to $A_{\theta}$ as the full axis of $\theta$ (with respect to $\Delta_{1}$ and $\Delta_{2}$ ). Note that since $\theta$ is a boundary loxodromic of $\Phi_{1} \cap \Phi_{2}$, it is primitive in $\Phi_{k}$ for both $k$ and its axis in $\Delta_{k}$ is precisely invariant under $\langle\theta\rangle$ in $\Phi_{k}$.

If $\theta$ is a primitive element of $\Gamma$, then its full axis $A_{\theta}$ is precisely invariant under $\langle\theta\rangle$ in $\Gamma$. However, if $\theta$ is not primitive in $\Gamma$, so that there exist some primitive $\gamma \in \Gamma$ and some integer $m \geq 2$ so that $\gamma^{m}=\theta$, this is not the case. It will always be that $A_{\theta} \cap \gamma\left(A_{\theta}\right)$ contains the fixed points of $\theta$, and it may also contain one or both of the components of the complement of the fixed points of $\theta$. However, $A_{\theta}$ still has the property that its stabilizer in $\Gamma$ is $\langle\theta\rangle$, and that it is precisely embedded under $\langle\theta\rangle$ in $\Gamma$, that is, no translate of $A_{\theta}$ by an element of $\Gamma$ separates $A_{\theta}$, even though they may intersect. 


\subsection{3-manifold basics}

We do not give here an introduction to the general theory of 3-manifolds, but instead restrict ourselves to those 3-manifolds which arise in this work. A 3-manifold $M$ is irreducible if every embedded 2-sphere in $M$ bounds a 3-ball. In particular, if $M$ is an irreducible 3-manifold which is not the 3-ball, then every component of $\partial M$ has positive genus. A 3-manifold $M$ is hyperbolizable if there exists a Kleinian group $\Gamma$ so that $M$ is homeomorphic to $\mathrm{M}_{\Gamma}=\left(\mathbf{H}^{3} \cup \Omega(\Gamma)\right) / \Gamma$; alternatively, we say that $M$ is uniformized by $\Gamma$. As $\Gamma$ acts freely on the contractible space $\mathbf{H}^{3} \cup \Omega(\Gamma)$ by orientation-preserving homeomorphisms, we see that $\mathrm{M}_{\Gamma}$ is necessarily orientable and irreducible.

We note here an equivalent formulation of convex co-compactness, in terms of the quotient 3manifold. As $\mathrm{M}_{\Gamma}$ is homeomorphic to $\mathrm{C}_{\Gamma}$ for a convex co-compact Kleinian group $\Gamma$ (see for example Epstein and Marden [8]), we have that $\Gamma$ is convex co-compact if and only if $\mathrm{M}_{\Gamma}$ is compact.

For a compact hyperbolizable 3 -manifold $M$, the choice of a uniformizing Kleinian group $\Gamma$ gives a bijection between $\Gamma$ and $\pi_{1}(M)$. Under this identification, the set of conjugacy classes of subgroups of the form $\pi_{1}(S)$, where $S$ is a component of $\partial M$, is in 1-1 correspondence with the set of stabilizers of components of $\Omega(\Gamma)$ which cover $S$.

\subsection{Surfaces in 3-manifolds}

A surface $S$ in a compact hyperbolizable 3-manifold $M$ is properly embedded if $S$ is compact and orientable, and if either $S \cap \partial M=\partial S$ or $S$ is contained in $\partial M$. A properly embedded surface $S$ in $M$ is incompressible if $S$ is not homeomorphic to the 2-sphere and if the inclusion $i: S \rightarrow M$ induces a monomorphism $i_{*}: \pi_{1}(S) \rightarrow \pi_{1}(M)$ on the level of fundamental groups. A 3-manifold is Haken if it contains an incompressible surface. It is known that a compact hyperbolizable 3-manifold with non-empty boundary is Haken (see for example Lemma 6.8 of Hempel [10]).

As a partial converse to this, it follows from Thurston's uniformization theorem for Haken 3-manifolds that a compact orientable irreducible Haken 3-manifold whose fundamental group contains no subgroup isomorphic to $\mathbf{Z} \oplus \mathbf{Z}$ can be uniformized by a convex co-compact Kleinian group. For a statement of Thurston's theorem in its full generality, and a discussion of its proof, the interested reader is referred to Morgan [20].

A compact hyperbolizable 3-manifold $M$ has incompressible boundary if each component of $\partial M$ is an incompressible surface in $M$, and has compressible boundary otherwise. It is an immediate consequence of the Loop Theorem (see for example Hempel [10]) and the Seifert-van Kampen Theorem that a compact hyperbolizable 3-manifold with non-abelian fundamental group has incompressible boundary if and only if its fundamental group does not admit a non-trivial splitting as a free product.

A surface $S$ in $M$ is non-peripheral if it is properly embedded and if the inclusion $i: S \rightarrow M$ is not homotopic to a map $f: S \rightarrow M$ for which $f(S) \subset \partial M$; if $\pi_{1}(S)$ is non-trivial, this is equivalent to requiring that the image $i_{*}\left(\pi_{1}(S)\right)$ in $\pi_{1}(M)$ is not conjugate to a subgroup of $\pi_{1}(T)$ for some component $T$ of $\partial M$. 
A surface $S$ in $M$ is essential if it is properly embedded, incompressible, and non-peripheral. We note that if $M$ is a compact hyperbolizable 3-manifold with non-empty boundary, then $M$ contains a non-separating essential surface $X$; this can be obtained by combining Lemmas 6.6 and 6.7 of Hempel [10]. We sometimes refer to such a surface as an embedded essential surface.

We also need a version of this notion for non-embedded closed surfaces. Say that a closed surface $S$ of positive genus in a compact hyperbolizable 3-manifold $M$ is an immersed essential surface if the inclusion $i: S \rightarrow M$ induces a monomorphism $i_{*}: \pi_{1}(S) \rightarrow \pi_{1}(M)$ on the level of fundamental groups and if the inclusion $i: S \rightarrow M$ is not homotopic to a map $f: S \rightarrow M$ with $f(S) \subset \partial M$; as above, the second condition is equivalent to requiring that the image $i_{*}\left(\pi_{1}(S)\right)$ in $\pi_{1}(M)$ is not conjugate to a subgroup of $\pi_{1}(T)$ for some component $T$ of $\partial M$.

\subsection{Connections between languages}

We now give some Lemmas which relate basic properties of a compact hyperbolizable 3-manifold to properties of its uniformizing convex co-compact Kleinian group. The first follows immediately from the Loop Theorem and Lemma 2.3 .

Lemma 2.4 Let $M$ be a compact hyperbolizable 3-manifold, uniformized by a convex co-compact Kleinian group $\Gamma$, and let $S$ be a component of $\partial M$ covered by a component $\Delta$ of $\Omega(\Gamma)$. Then, $\operatorname{st}_{\Gamma}(\Delta)$ is quasifuchsian if and only if $S$ is incompressible.

As an immediate consequence of Lemma 2.4, we see that $\partial M$ is incompressible if and only if $\Lambda(\Gamma)$ is connected. So, if $\Gamma$ is a non-elementary convex co-compact Kleinian group, then it admits a non-trivial splitting as a free product if and only if some component subgroup is not quasifuchsian. Also, if $M$ is a compact hyperbolizable 3-manifold with non-empty incompressible boundary, uniformized by a convex co-compact Kleinian group, then each component of $\partial M$ has genus at least two.

The second Lemma gives a way of detecting immersed essential surfaces.

Lemma 2.5 Let $M$ be a compact hyperbolizable 3-manifold with non-empty boundary, uniformized by a convex co-compact Kleinian group $\Gamma$. If $S$ is a closed immersed incompressible surface in $M$, then $\Phi=\pi_{1}(S)$ is a quasifuchsian subgroup of $\Gamma$; conversely, if $\Phi$ is a quasifuchsian subgroup of $\Gamma$, there exists a closed immersed incompressible surface $S$ in $M$ with $\pi_{1}(S)=\Phi$. Moreover, $S$ is essential if and only if $\Phi$ is not contained in a component subgroup of $\Gamma$.

Proof Since $M$ has non-empty boundary, the domain of discontinuity of $\Gamma$ is non-empty, and so $\Phi$ is necessarily convex co-compact. The fact that $\Phi$ is quasifuchsian follows immediately from Lemma 2.2 and the assumption that $S$ is incompressible. For the converse, if $D$ is any disc in $\mathbf{H}^{3}$ which is invariant under $\Phi$, then the image of $D$ in $M$ is the desired surface; for example, we may take $D$ to be one of the two components of the boundary of the convex hull $\mathrm{CH}_{\Phi}$ of $\Phi$ in $\mathbf{H}^{3}$. The last statement follows from the definition of essential. 


\subsection{Essential annuli}

Let $M$ be a compact hyperbolizable 3-manifold with incompressible boundary, uniformized by a convex co-compact Kleinian group $\Gamma$. We describe here how it is possible to detect an embedded essential annulus $A$ in $M$ by examining the intersection of the fundamental groups of the boundary components of $M$ which contain the end curves of $A$. As the identification of $\pi_{1}(M)$ with $\Gamma$ gives a bijective correspondence between the set of conjugacy classes of the fundamental groups of all boundary components of $M$ and the set of component subgroups of $\Gamma$, this is equivalent to examining the intersection of pairs of component subgroups of $\Gamma$. This in turn is equivalent to examining the intersection of the limit sets of the component subgroups of $\Gamma$. An explicit description of these equivalences is given in the following Lemma.

Lemma 2.6 Let $M$ be a compact hyperbolizable 3-manifold with incompressible boundary, uniformized by a convex co-compact Kleinian group $\Gamma$. Then, there exists an embedded essential annulus $A$ in $M$ if and only if there exist two components $\Delta_{1}$ and $\Delta_{2}$ of $\Omega(\Gamma)$ whose closures have non-empty intersection.

Proof Let $A$ be an essential annulus $A$ in $M$ and lift to the universal cover $\mathbf{H}^{3} \cup \Omega(\Gamma)$ of $M$. Since $A$ is incompressible and has fundamental group isomorphic to $\mathbf{Z}$, the interior of $A$ lifts to an open disc $D$ in $\mathbf{H}^{3}$ which is invariant under a loxodromic cyclic subgroup $\langle\gamma\rangle$ of $\Gamma$. The boundary of $A$ lifts to a pair of arcs $a_{1}$ and $a_{2}$ in $\Omega(\Gamma)$, both of which are invariant under $\langle\gamma\rangle$. Since $A$ is essential, these two $\operatorname{arcs} a_{1}$ and $a_{2}$ necessarily lie in different components $\Delta_{1}$ and $\Delta_{2}$ of $\Omega(\Gamma)$. The fixed points of $\gamma$ lie in the closures of both $a_{1}$ and $a_{2}$, and so the closures of $\Delta_{1}$ and $\Delta_{2}$ have non-empty intersection.

Suppose now that there exist two components $\Delta_{1}$ and $\Delta_{2}$ of $\Omega(\Gamma)$ whose closures have non-trivial intersection. Let $\Phi_{k}$ be the stabilizer of $\Delta_{k}$ in $\Gamma$, and note that the assumption of incompressible boundary implies that both $\Phi_{1}$ and $\Phi_{2}$ are quasifuchsian. By the discussion in Section 2.3, there exists a boundary loxodromic $\theta$ in $\Phi_{1} \cap \Phi_{2}$ whose full axis $A_{\theta}$ is precisely embedded under $\langle\theta\rangle$ in $\Gamma$. By Theorem VII.B.16 of Maskit [15], there exists an open disc $D$ in $\mathbf{H}^{3}$ which is precisely invariant under $\langle\theta\rangle$ in $\Gamma$ and whose boundary in $\mathbf{H}^{3} \cup \overline{\mathbf{C}}$ is equal to $A_{\theta}$. Removing the fixed points of $\theta$ from the closed disc $D \cup A_{\theta}$ and projecting to $M$, we get an embedded annulus in $M$. This annulus is necessarily essential, as $A_{\theta}$ separates $\Lambda(\Gamma)$.

As is mentioned in Section 2.3, it follows from Theorem 3 of Maskit [14] that there exist two components $\Delta_{1}$ and $\Delta_{2}$ of $\Omega(\Gamma)$ whose closures have non-empty intersection if and only if there exist two component subgroups $\Phi_{1}$ and $\Phi_{2}$ in $\Gamma$ which have non-trivial intersection. Hence, we have an alternative phrasing of Lemma 2.6, which is that there exists an essential annulus in $M$ if and only if there exist two component subgroups of $\Gamma$ whose intersection is non-trivial. 


\subsection{Acylindrical 3-manifolds}

Let $M$ be a compact hyperbolizable 3-manifold, and let $S_{1}, \ldots, S_{p}$ be a collection of components of $\partial M$. The subset $\mathcal{S}=S_{1} \cup \cdots \cup S_{p}$ of $\partial M$ is an-annular if each $S_{j}$ is incompressible and if there does not exist an embedded essential annulus in $M$ both of whose boundary curves lie in $\mathcal{S}$. A compact hyperbolizable 3-manifold $M$ is acylindrical if its entire boundary $\partial M$ is an-annular.

The following Lemma follows immediately from Lemma 4.2 of Morgan [20].

Lemma 2.7 Let $M$ be a compact hyperbolizable acylindrical 3-manifold, and let $X$ be a properly embedded incompressible non-separating surface in $M$. Suppose that among all such surfaces $X$ has maximimal Euler characteristic and minimal genus given its Euler characteristic. Then, for each component $S$ of $\partial M$, the intersection $\pi_{1}(X) \cap \pi_{1}(S)$ is either trivial or infinite cyclic.

In terms of the uniformizing Kleinian group $\Gamma$, Lemma 2.7 can be phrased as follows. Let $\Theta$ be a choice of conjugacy class of the fundamental group of $X$ in $\Gamma$, and for each component $S$ of $\partial M$, let $\Phi$ be a choice of conjugacy class of the fundamental group of $S$ in $\Gamma$. Then, the intersection $\Theta \cap \Phi$ is either trivial or loxodromic cyclic.

The following Lemma follows immediately from Lemma 2.6.

Lemma 2.8 Let $M$ be a compact hyperbolizable 3-manifold with incompressible boundary, uniformized by a convex co-compact Kleinian group $\Gamma$. Then, $M$ is acylindrical if and only if the closures of each pair of distinct components of $\Omega(\Gamma)$ are disjoint.

\section{Combination theorems and graphs of groups}

One of the tools often used to construct Kleinian groups, and to obtain specific information about the behavior of the Kleinian groups constructed, are the Klein-Maskit combination theorems. We give in this Section a brief sketch of the particular case of the combination theorems used in this work, and we also frame its statement in the general language of the Bass-Serre theory of fundamental groups of graphs of groups. For a detailed discussion of the combination theorems, the interested reader is referred to Maskit [15], and for a detailed discussion of Bass-Serre theory, the interested reader is referred to Serre [25] or to Scott and Wall [23].

The first step is to describe the classical Klein-Maskit combination theorem for the amalgamated free product of two Kleinian groups along a finitely generated subgroup, often referred to in the literature as the Klein-Maskit combination of type I. In this paper, we are interested in only one small part of the combination of type I, namely giving topological conditions on a pair of Kleinian groups $\Phi_{1}$ and $\Phi_{2}$ which imply that the group $\Gamma=\left\langle\Phi_{1}, \Phi_{2}\right\rangle$ is a Kleinian group isomorphic to the amalgamated free product of $\Phi_{1}$ and $\Phi_{2}$ along $\Xi=\Phi_{1} \cap \Phi_{2}$. These topological conditions are given in terms of a Jordan curve which behaves well under the action of $\Phi_{1}, \Phi_{2}$, and $\Xi$, and which is then seen to behave well under the action of $\Gamma$. 
Theorem 3.1 Let $\Phi_{1}$ and $\Phi_{2}$ be finitely generated Kleinian groups whose intersection $\Xi=\Phi_{1} \cap \Phi_{2}$ is finitely generated and has infinite index in both $\Phi_{1}$ and $\Phi_{2}$. Assume there exists a Jordan curve $c$ determining closed discs $E_{1}$ and $E_{2}$ in $\overline{\mathbf{C}}$ so that $c$ segregates $\Lambda\left(\Phi_{1}\right)$ from $\Lambda\left(\Phi_{2}\right)$, so that $E_{k}$ is precisely invariant under $\Xi$ in $\Phi_{k}$, and so that $E_{k} \cap \Lambda\left(\Phi_{k}\right)=\Lambda(\Xi)$ for both $k$.

Then, the group $\Gamma$ generated by $\Phi_{1}$ and $\Phi_{2}$ is a Kleinian group isomorphic to $\Phi_{1} *_{\Xi} \Phi_{2}$. Moreover, we have that $c \cap \Lambda(\Gamma)=\Lambda(\Xi)$ and that $c$ is precisely invariant under $\Xi$ in $\Gamma$.

We pause here to note several facts about the relationship between $\Phi_{1}, \Phi_{2}$, and $\Xi$ implied by the hypotheses of Theorem 3.1. Since $c$ segregates $\Lambda\left(\Phi_{1}\right)$ from $\Lambda\left(\Phi_{2}\right)$, we have that $c$ separates $\Lambda(\Gamma)$. Combining this with the assumption that $E_{j}$ is precisely invariant under $\Xi_{j}$ in $\Phi_{j}$, we see that $\Lambda\left(\Phi_{j}\right)$ lies in $E_{3-j}$. Since $\Xi$ is a finitely generated infinite index subgroup of $\Phi_{k}$, and since $\Phi_{k}$ has non-empty domain of discontinuity, Lemma 2.1 implies that $\Lambda(\Xi)$ is a proper subset of $\Lambda\left(\Phi_{k}\right)$.

We now set Theorem 3.1 in the general framework of the Bass-Serre theory of fundamental groups of graphs of groups. After giving a brief sketch of the Bass-Serre theory sufficient for the purposes of this work, we describe Theorem 3.1 in this language, and then describe an extension of Theorem 3.1 which allows combination along a system of several Jordan curves simultaneously.

A graph $T$ consists of a pair $(V, E)$, where $V$ is a finite set consisting of the vertices of $T$, and $E$ is a subset of $V \times V$ consisting of the (directed) edges of $T$. Given an edge $y=(o(y), t(y)) \in E$, let $\bar{y}$ be the inverse edge $(t(y), o(y))$; we assume that $E$ is closed under the taking of inverses. A tree is a graph which contains no cycles.

A graph of groups $(G, T)$ consists of a graph $T$ and a collection $G$ of groups, together with the assignment of a group $G_{p} \in G$ to each vertex $p \in V$, a group $H_{y} \in G$ to each edge $y \in E$, subject to the condition that $H_{y}=H_{\bar{y}}$, and monomorphisms $H_{y} \rightarrow G_{t(y)}$ for all $y \in E$, which we denote $a \mapsto a^{y}$.

Define $F(G, T)$ to be the abstract group generated by the elements of the groups $G_{p}$ for all $p \in V$ and the elements $y$ of $E$, subject to the relations $\bar{y}=y^{-1}$ and $y a^{y} y^{-1}=a^{\bar{y}}$ for $y \in E$ and $a \in H_{y}$. In the case that $T$ is a tree, $F(G, T)$ is the fundamental group of the graph of groups $(G, T)$.

The essential consequence of this construction is that $F(G, T)$ is built up by independently taking the amalgamated free products of the vertex groups along their common subgroups, which are the edge groups. In the case that $T$ is a tree, $F(G, T)$ splits as an amalgamated free product along any of the edge groups $H_{y}$.

In the case of Theorem 3.1, the graph $T$ consists of two vertices $v_{1}$ and $v_{2}$ joined by a single edge $y$. The groups $\Phi_{1}$ and $\Phi_{2}$ are the groups associated to the vertices, the group $\Xi=\Phi_{1} \cap \Phi_{2}$ is associated to the edge, and the monomorphisms from $\Xi$ to the $\Phi_{k}$ are the identity. Theorem 3.1 can then be described as giving topological conditions on $\Phi_{1}, \Phi_{2}$, and $\Xi$ under which the group $\left\langle\Phi_{1}, \Phi_{2}\right\rangle$ is equal to the fundamental group of the graph of groups $F(G, T)$.

In order to better describe the extension of Theorem 3.1 to more than two groups, we need an extension of the notion of a precisely invariant set. Given a Kleinian group $\Gamma$, a collection $\left(X_{1}, \ldots, X_{p}\right)$ of subsets of $\mathbf{H}^{3} \cup \overline{\mathbf{C}}$, and a collection $\left(\Phi_{1}, \ldots, \Phi_{p}\right)$ of subgroups of $\Gamma$, say that the 
collection $\left(X_{1}, \ldots, X_{p}\right)$ is precisely invariant under $\left(\Phi_{1}, \ldots, \Phi_{p}\right)$ in $\Gamma$ if for each $\gamma \in \Gamma, \gamma\left(X_{j}\right) \cap X_{k}$ is non-empty if and only if $j=k$ and $\gamma \in \Phi_{j}$; that is, each $X_{j}$ is precisely invariant under $\Phi_{j}$ in $\Gamma$, and $X_{j} \cap \gamma\left(X_{k}\right)$ is empty for all $\gamma \in \Gamma$ and $j \neq k$. By way of example, any collection of inequivalent components of the domain of discontinuity of a Kleinian group $\Gamma$ is precisely invariant under their stabilizers in $\Gamma$, while if $\Gamma$ is convex co-compact and uniformizes an acylindrical 3-manifold, any collection of closures of inequivalent components is precisely invariant under their stabilizers in $\Gamma$.

Multiple combination theorems, such as the one described below, have been considered in some detail by Maskit, see especially [17] and [13]. We are interested only in a special case of multiple combination. In order to best describe it, we use the language of the Bass-Serre theory.

Let $T_{n}$ denote the tree which is the cone on $n$ points $p_{1}, \ldots, p_{n}$, with cone point $p_{0}$, and label the edges of $T_{n}$ as $e_{1}, \ldots, e_{n}$, where $e_{j}$ joins $p_{0}$ to $p_{j}$. Given finitely generated groups $\Theta, \Phi_{1}, \ldots, \Phi_{n}$, associate $\Theta$ with $p_{0}$, associate $\Phi_{j}$ with $p_{j}$, and associate $\Theta \cap \Phi_{j}$ with the edge $e_{j}$, where the monomorphisms from $\Theta \cap \Phi_{j}$ to $\Theta$ and to $\Phi_{j}$ are both the identity. We denote this graph of groups by $G\left(\Theta ; \Phi_{1}, \ldots, \Phi_{n}\right)$.

In the case that $\Theta, \Phi_{1}, \ldots, \Phi_{n}$ are Kleinian groups, the multiple combination theorem stated below gives topological conditions, in terms of a well-behaved collection of Jordan curves, which guarantee that $\left\langle\Theta, \Phi_{1}, \ldots, \Phi_{n}\right\rangle$ is isomorphic to the fundamental group of the graph of groups $G\left(\Theta ; \Phi_{1}, \ldots, \Phi_{n}\right)$. The proof of Theorem 3.2 follows immediately by successive applications of Theorem 3.1.

Theorem 3.2 Let $\Theta, \Phi_{1}, \ldots, \Phi_{n}$ be finitely generated Kleinian groups so that each $\Xi_{j}=\Phi_{j} \cap \Theta$ is finitely generated and has infinite index in both $\Phi_{j}$ and $\Theta$. Let $c_{1}, \ldots, c_{n}$ be a collection of disjoint Jordan curves in $\overline{\mathbf{C}}$ bounding a connected domain $D$ in $\overline{\mathbf{C}}$, and let $D_{j}$ and $E_{j}$ be the closed discs determined by $c_{j}$, labelled so that $D_{j}$ contains $D$. Suppose that $c_{j}$ segregates $\Lambda(\Theta)$ from $\Lambda\left(\Phi_{j}\right)$, that $c_{j} \cap \Lambda\left(\Phi_{j}\right)=c_{j} \cap \Lambda(\Theta)=\Lambda\left(\Xi_{j}\right)$ for each $j$, that $D_{j}$ is precisely invariant under $\Xi_{j}$ in $\Phi_{j}$, and that the collection $\left(E_{1}, \ldots, E_{n}\right)$ is precisely invariant under $\left(\Xi_{1}, \ldots, \Xi_{n}\right)$ in $\Theta$.

Then, the group $\Gamma=\left\langle\Theta, \Phi_{1}, \ldots, \Phi_{n}\right\rangle$ is a Kleinian group isomorphic to the fundamental group of the graph of groups $G\left(\Theta ; \Phi_{1}, \ldots, \Phi_{n}\right)$. Moreover, $c_{j} \cap \Lambda(\Gamma)=\Lambda\left(\Xi_{j}\right)$ for each $j$, and the collection $\left(c_{1}, \ldots, c_{n}\right)$ is precisely invariant under $\left(\Xi_{1}, \ldots, \Xi_{n}\right)$ in $\Gamma$.

Note that the hypotheses of Theorem 3.2 are saying that $\Theta, \Phi_{j}$, and $c_{j}$ satisfy the hypotheses of Theorem 3.1 for each $j$, and that $c_{j}$ and $c_{k}$ don't interfere with each other for $j \neq k$. Also, as above, note that the hypotheses of Theorem 3.2 imply that $\Lambda(\Theta)$ lies in the closure $\bar{D}$ of $D$ and that $\Lambda\left(\Phi_{j}\right)$ lies in $E_{j}$, and that each $c_{j}$ separates $\Lambda(\Gamma)$.

\section{The proofs}

We are now ready to prove the main results of this paper, namely Proposition 4.1 and Theorem 4.2. Before doing so, we briefly describe the situation. We are given a compact hyperbolizable acylindrical 3-manifold $M$ whose boundary is incompressible and is the non-empty union of surfaces each of genus at least two, so that $M$ can be uniformized by a convex co-compact Kleinian group 
$\Gamma=\pi_{1}(M)$ with connected limit set. We also have an embedded essential surface $X$ in $M$. If $X$ is closed, there is nothing to do.

If not, let $\Theta$ be the fundamental group of $X$ in $\Gamma$, write $\partial X$ as the union of a collection $a_{1}, \ldots, a_{n}$ of simple closed curves, and for each component $a_{j}$ of $\partial X$, let $\Phi_{j}$ be the subgroup of $\Gamma$ which is the fundamental group of the component $S_{j}$ of $\partial M$ containing $a_{j}$; we note that $\Phi_{j}$ and $\Phi_{k}$ will be conjugate in $\Gamma$, though not in $\Theta$, if $a_{j}$ and $a_{k}$ lie in the same component of $\partial M$. Consider the subgroup $\Gamma^{o}$ of $\Gamma$ which is generated by $\Theta, \Phi_{1}, \ldots, \Phi_{n}$. One might hope that $\Gamma^{o}$ would be the fundamental group of the graph of groups $G\left(\Theta ; \Phi_{1}, \ldots, \Phi_{n}\right)$ as described above, representing the fundamental group of the regular neighborhood $N$ of the 2-complex constructed from the surfaces $X, S_{1}, \ldots, S_{n}$ by gluing $X$ to $S_{j}$ along $a_{j}$. If so, the fundamental group of $N$ would inject into $\Gamma$. In particular, the boundary components of $N$, at least one of which is non-peripheral, would be incompressible.

Unfortunately, this picture does not hold in general. The boundary components of $N$ can be compressible. However, if we allow ourselves the possibility of passing to finite index subgroups $\Phi_{j}^{o}$ of the $\Phi_{j}$, we can arrange for the subgroup generated by $\Theta$ and the $\Phi_{j}^{o}$ to be a graph of groups as desired. This is the content of the following Proposition, though the Proposition is proven in slightly more generality. This subgroup represents the fundamental group of a thickened 2-complex $\tilde{N}$ which maps $\pi_{1}$-injectively to $M$. Some of the boundary components of $\tilde{N}$ naturally cover components of $\partial M$, but there is at least one component of $\partial \tilde{N}$ which does not cover a component of $\partial M$, and so gives rise to an immersed essential surface in $M$.

Proposition 4.1 Let $M$ be a compact hyperbolizable 3-manifold with non-empty boundary, uniformized by a convex co-compact Kleinian group $\Gamma$. Let $\Theta$ be a finitely generated subgroup of $\Gamma$, and let $\Delta_{1}, \ldots, \Delta_{n}$ be a collection of components of $\Omega(\Gamma)$ which are inequivalent under $\Theta$ and which cover an an-annular collection of components of $\partial M$. Set $\Phi_{j}=\operatorname{st}_{\Gamma}\left(\Delta_{j}\right)$, and assume that $\Xi_{j}=\Theta \cap \Phi_{j}$ has infinite index in both $\Phi_{j}$ and $\Theta$ for all $1 \leq j \leq n$.

Then, for each $j$ there exists a finite index subgroup $\Phi_{j}^{o}$ of $\Phi_{j}$ so that $\Theta \cap \Phi_{j}^{o}=\Xi_{j}$ and so that the group $\Gamma^{o}=\left\langle\Theta, \Phi_{1}^{o}, \ldots, \Phi_{n}^{o}\right\rangle$ is isomorphic to the fundamental group of the graph of groups $G\left(\Theta ; \Phi_{1}^{o}, \ldots, \Phi_{n}^{o}\right)$.

Proof We proceed by showing that the hypotheses of Theorem 3.2 hold. By Lemma 2.4, each $\Phi_{j}$ is quasifuchsian, as it covers an incompressible component of $\partial M$; also, since $\Omega(\Gamma)$ is non-empty, $\Theta$ is convex co-compact. We now apply Theorem 2 of Susskind [27] to conclude that $\partial \Delta_{j} \cap \Lambda(\Theta)=\Lambda\left(\Phi_{j}\right) \cap \Lambda(\Theta)=\Lambda\left(\Xi_{j}\right)$ and that $\Xi_{j}$ is finitely generated, and hence convex co-compact, for each $1 \leq j \leq n$. Let $\pi: \Omega(\Theta) \rightarrow S=\Omega(\Theta) / \Theta$ denote the covering map.

We now proceed to construct the collection $c_{1}, \ldots, c_{n}$ of Jordan curves satisfying the hypotheses of Theorem 3.2. Since $\Delta_{1}, \ldots, \Delta_{n}$ cover an an-annular collection of components of $\partial M$, we see that the collection of their closures $\left(\overline{\Delta_{1}}, \ldots, \overline{\Delta_{n}}\right)$ is precisely invariant under $\left(\Phi_{1}, \ldots, \Phi_{n}\right)$ in $\Gamma$; in particular, each $\overline{\Delta_{j}}$ is precisely invariant under $\Phi_{j}$ in $\Gamma$, and hence is precisely invariant under $\Xi_{j}=\Theta \cap \Phi_{j}$ in $\Theta$. The Jordan curve $c_{j}$ will be a perturbation of $\partial \Delta_{j}$. 
Let $\widetilde{Y_{j}}=\overline{\Delta_{j}}-\Lambda\left(\Xi_{j}\right)$. Since $\widetilde{Y_{j}}$ is contained in $\Omega(\Theta)$, its image $Y_{j}=\pi\left(\widetilde{Y_{j}}\right)$ in $S$ is an embedded subsurface of $S$. Since $\Xi_{j}$ is finitely generated and of infinite index in $\Phi_{j}, Y_{j}$ is compact and has non-empty boundary. Note that $\Xi_{j}$ may be trivial, in which case $Y_{j}$ is a closed disc in $S$.

Note that $Y_{j}$ and $Y_{k}$ are disjoint for $j \neq k$. For if not, there exists some point $x \in Y_{j} \cap Y_{k}$. Since the interiors of $Y_{j}$ and $Y_{k}$ are covered by components of $\Omega(\Gamma)$ which are inequivalent under $\Theta$, they are disjoint, and so $x$ must lie in $\partial Y_{j} \cap \partial Y_{k}$. Lifting back to $\Omega(\Gamma)$, there are components $\Delta_{j}^{\prime}$ and $\Delta_{k}^{\prime}$, equivalent under the action of $\Theta$ to $\Delta_{j}$ and $\Delta_{k}$ respectively, covering the interiors of $Y_{j}$ and $Y_{k}$, so that $x$ lies in $\partial \Delta_{j}^{\prime} \cap \partial \Delta_{k}^{\prime}$, contrary to the assumption that $\Delta_{1}, \ldots, \Delta_{n}$ cover an an-annular collection of components of $\partial M$.

As $Y_{1}, \ldots, Y_{n}$ are disjoint subsets of $S$, there exists some $\varepsilon>0$ so that the closed $\varepsilon$-neighborhoods $Y_{1}^{\prime}, \ldots, Y_{n}^{\prime}$ of $Y_{1}, \ldots, Y_{n}$ on $S$ are disjoint and have the property that $Y_{j}^{\prime}$ is homeomorphic to $Y_{j}$ for each $j$. The reason for using $\varepsilon$-neighborhoods of the $Y_{j}$ is to push the boundaries of the lifts of the $Y_{j}$ 's off of the rest of the limit sets of the $\Phi_{j}$. Specifically, let $E_{j}$ be the closure of the lift of $Y_{j}^{\prime}$ to $\overline{\mathbf{C}}$ whose interior contains $\Delta_{j}$, and note that $E_{j}$ is a closed topological disc. The boundary $c_{j}$ of $E_{j}$ is a Jordan curve which is invariant under $\Xi_{j}$ and which intersects $\Lambda(\Theta)$ exactly in $\Lambda\left(\Xi_{j}\right)$. In fact, $c_{j}$ is a $\Xi_{j}$-equivariant perturbation of $\partial \Delta_{j}$ which is homotopic to $\partial \Delta_{j}$ relative to $\Lambda\left(\Xi_{j}\right)$. Note that $c_{1}, \ldots, c_{n}$ bound a connected region in $\overline{\mathbf{C}}$.

Since $\Lambda\left(\Phi_{j}\right)$ lies in $\Delta_{j}$, which is contained in $E_{j}$, we see that $c_{j}$ does not separate $\Lambda\left(\Phi_{j}\right)$. Also, since the interior of $E_{j}$ lies in $\Omega(\Theta), c_{j}$ does not separate $\Lambda(\Theta)$; in particular, each $c_{j}$ segregates $\Lambda(\Theta)$ from $\Lambda\left(\Phi_{j}\right)$. Since $E_{1}, \ldots, E_{n}$ are constructed from disjoint subsurfaces of $S$, we see that the collection $\left(E_{1}, \ldots, E_{n}\right)$ of closed discs is precisely invariant under $\left(\Xi_{1}, \ldots, \Xi_{n}\right)$ in $\Theta$.

Let $D_{j}$ be the closed disc determined by $c_{j}$ complementary to $E_{j}$; since $E_{j}$ is invariant under $\Xi_{j}$, so is $D_{j}$. In order to satisfy the hypotheses of Theorem 3.2, it remains only to show, for each $1 \leq j \leq n$, that there exists a finite index subgroup $\Phi_{j}^{o}$ of $\Phi_{j}$ containing $\Xi_{j}$, so that $D_{j}$ is precisely invariant under $\Xi_{j}$ in $\Phi_{j}^{o}$. In order to find such a subgroup, we use the main Theorem of Scott [22], which states that the fundamental group $G$ of a closed orientable surface is LERF (locally extended residually finite), which means that given a finitely generated subgroup $H$ of $G$ and an element $g \in G-H$, there exists a finite index subgroup $G^{o}$ of $G$ containing $H$ and not $g$. In our situation, this Theorem can be phrased as saying that if $g \in \Phi_{j}-\Xi_{j}$ is any element, then there exists a finite index subgroup $\Phi_{j}^{o}$ of $\Phi_{j}$ which contains $\Xi_{j}$ and does not contain $g$. Moreover, if we consider the double coset decomposition $\Phi_{j}=\bigcup_{q=0}^{\infty} \Xi_{j} \cdot \varphi_{q} \cdot \Xi_{j}$ of $\Phi_{j}$ with respect to $\Xi_{j}$, we see that some element of the double coset $\Xi_{j} \cdot \varphi_{q} \cdot \Xi_{j}$ lies in the subgroup $\Phi_{j}^{o}$ if and only if every element of $\Xi_{j} \cdot \varphi_{q} \cdot \Xi_{j}$ lies in $\Phi_{j}^{o}$. Hence, for each $1 \leq j \leq n$, it suffices to show that only finitely many double cosets of $\Xi_{j}$ in $\Phi_{j}$ contain an element $\psi$ for which $D_{j} \cap \psi\left(D_{j}\right)$ is non-empty.

So, write $D_{j}=\Lambda\left(\Xi_{j}\right) \cup Z$, where $Z=D_{j}-\Lambda\left(\Xi_{j}\right)$. We first consider $Z$; note that $Z / \Xi_{j}$ is compact, as it is homeomorphic to $Y_{j}$. Let $F$ be a compact fundamental domain for the action of $\Xi_{j}$ on $Z$, suppose that there exists some $\varphi \in \Phi_{j}$ for which $Z \cap \varphi(Z)$ is non-empty, and let $\Xi_{j} \cdot \varphi_{q} \cdot \Xi_{j}$ be the double coset containing $\varphi$. Then, there exists some $\varphi^{\prime}$ in $\Xi_{j} \cdot \varphi_{q} \cdot \Xi_{j}$ so that $F \cap \varphi^{\prime}(F)$ is non-empty. To see this, choose a point $x \in Z \cap \varphi(Z)$. Then, there exist $\xi \in \Xi_{j}$ so that $x \in \xi(F)$ and $\xi^{\prime} \in \Xi_{j}$ so that $x \in \varphi \cdot \xi^{\prime}(F)$, which implies that $F \cap \xi^{-1} \cdot \varphi \cdot \xi^{\prime}(F)$ is non-empty. Since $\Phi_{j}$ acts 
properly discontinuously on $\Omega\left(\Phi_{j}\right)$ and $Z$ lies in $\Omega\left(\Phi_{j}\right)$, there can exist only finitely many elements of $\psi$ of $\Phi_{j}$ for which $F \cap \psi(F)$ is non-empty, and so only finitely many double cosets of $\Xi_{j}$ in $\Phi_{j}$ can contain an element $\varphi$ for which $Z \cap \varphi(Z)$ is non-empty. By Scott's Theorem, there exists a finite index subgroup $\Phi_{j}^{o o}$ of $\Phi_{j}$ containing $\Xi_{j}$, so that $Z$ is precisely invariant under $\Xi_{j}$ in $\Phi_{j}^{o o}$.

We now show that only finitely many double cosets of $\Xi_{j}$ in $\Phi_{j}^{o o}$ can contain an element $\varphi$ for which $D_{j} \cap \varphi\left(D_{j}\right)$ is non-empty. For each such $\varphi$, we have that $\Lambda\left(\Xi_{j}\right) \cap \varphi\left(\Lambda\left(\Xi_{j}\right)\right)$ is non-empty; since $\Lambda\left(\Xi_{j}\right) \cap \varphi\left(\Lambda\left(\Xi_{j}\right)\right)=\Lambda\left(\Xi_{j} \cap \varphi \cdot \Xi_{j} \cdot \varphi^{-1}\right)$, again by Theorem 2 of Susskind [27], we see that $\Xi_{j} \cap \varphi \cdot \Xi_{j} \cap \cdot \varphi^{-1}$ is non-trivial. If $\varphi$ is not in the same double coset as the identity, then $\pi(Z)$ and $\pi(\varphi(Z))$ are disjoint subsurfaces on $S$. Since $\pi(Z)$ is compact, it has only finitely many boundary components, and so there can be only finitely many double cosets of $\Xi_{j}$ in $\Phi_{j}^{o o}$ containing such $\varphi$. Hence, again by Scott's Theorem, there exists a finite index subgroup $\Phi_{j}^{o}$ of $\Phi_{j}$ which contains $\Xi_{j}$, so that $D_{j}$ is precisely invariant under $\Xi_{j}$ in $\Phi_{j}^{o}$.

We have now shown that all the hypotheses of Theorem 3.2 are satisfied, and so the group $\Gamma^{o}=\left\langle\Theta, \Phi_{1}^{o}, \ldots, \Phi_{n}^{o}\right\rangle$ is a Kleinian group isomorphic to the fundamental group of the graph of groups $G\left(\Theta ; \Phi_{1}^{o}, \ldots, \Phi_{n}^{o}\right)$.

The proof of Theorem 4.2 is now an easy application of Proposition 4.1, together with the discussion of Section 2.

Theorem 4.2 Let $M$ be a compact hyperbolizable acylindrical 3-manifold with non-empty incompressible boundary, in which every boundary component has genus at least two. Then, $M$ contains a closed immersed essential surface.

Proof Note that the hypotheses imply that $M$ is uniformized by a convex co-compact Kleinian group $\Gamma$. Let $X$ be a surface as in the statement of Lemma 2.7, that is, $X$ is a non-separating essential surface which maximizes Euler characteristic and minimizes genus among all such surfaces; set $\Theta=\pi_{1}(X)$. If $X$ is closed, we are done. Suppose not, and let $a_{1}, \ldots, a_{p}$ be an enumeration of the components of $\partial X$. For each $j$, let $S_{j}$ be the component of $\partial M$ containing $a_{j}$, and note that $\Phi_{1}=\pi_{1}\left(S_{1}\right), \ldots, \Phi_{p}=\pi_{1}\left(S_{p}\right)$ are the component subgroups of a collection of distinct components of $\Omega(\Gamma)$ which are inequivalent under $\Theta$. By Lemma 2.7, each $\Xi_{j}=\Theta \cap \Phi_{j}$ is loxodromic cyclic.

Proposition 4.1 yields the existence of finite index subgroups $\Phi_{j}^{o}$ of $\Phi_{j}$ containing $\Xi_{j}$ for $1 \leq$ $j \leq p$ so that the group $\Gamma^{o}=\left\langle\Theta, \Phi_{1}^{o}, \ldots, \Phi_{p}^{o}\right\rangle$ is the fundamental group of the graph of groups $G\left(\Theta, \Phi_{1}^{o}, \ldots, \Phi_{p}^{o}\right)$.

It still remains to show that $\Gamma^{o}$ has infinite index in $\Gamma$ and that $\Gamma^{o}$ does not split as a free product. Assuming these, we complete the proof. Since $\Gamma^{o}$ does not split as a free product, Lemma 2.4 implies that every component subgroup of $\Gamma^{o}$ is quasifuchsian. Since $\Gamma^{o}$ has infinite index in $\Gamma$, Lemma 2.1 implies that $\Lambda\left(\Gamma^{o}\right)$ is a proper subset of $\Lambda(\Gamma)$, and so there is a quasifuchsian component subgroup $\Phi$ of $\Gamma^{o}$ which is not contained in a component subgroup of $\Gamma$. By Lemma 2.5, there exists a closed immersed essential surface in $M$ associated to $\Phi$, and we are done. 
To see that $\Gamma^{o}$ does not split as a free product, let $S_{j}^{o}$ be the finite cover of $S_{j}$ associated to $\Phi_{j}^{o}$, and note that Proposition 4.1 implies that $a_{j}$ lifts to a closed curve, which we again call $a_{j}$, on $S_{j}^{o}$. Consider the 2-complex $Y$ formed from the surfaces $X, S_{1}^{o}, \ldots, S_{p}^{o}$ by attaching $X$ to $S_{j}^{o}$ along $a_{j}$, and note that $\Gamma^{o}$ is the fundamental group of this 2-complex $Y$. In order to show that $\Gamma^{o}$ does not split as a free product, it suffices to show that $\Gamma^{o}$ has one end, which is equivalent to showing that no compact set separates the universal cover $\widetilde{Y}$ of $Y$; for a discussion of this equivalence, as well as the relationship between ends and free product splittings in general, see for example Stallings [26].

That no compact set separates $\widetilde{Y}$ follows immediately from the description of $\widetilde{Y}$. Let $\widetilde{X}$ be the universal cover of $X$, and note that $\tilde{X}$ is homeomorphic to the complement of a union of disjoint open half spaces in the hyperbolic plane $\mathbf{H}^{2}$; moreover, the boundary components of $\widetilde{X}$ cover the boundary $a_{1} \cup \cdots \cup a_{n}$ of $X$. The universal cover $\widetilde{S_{j}}$ of $S_{j}^{o}$ is homeomorphic to the hyperbolic plane $\mathbf{H}^{2}$, and the lift of $a_{j}$ to $S_{j}^{o}$ is a collection of disjoint lines in $\widetilde{S_{j}}$. We can construct $\tilde{Y}$ by taking infinitely many copies of each of $\widetilde{X}, \widetilde{S_{1}}, \ldots, \widetilde{S_{n}}$ and attaching them appropriately. It is easy to see that no compact subset of $\tilde{Y}$ can separate $\tilde{Y}$.

The fact that $\Gamma^{o}$ has infinite index in $\Gamma$ is now immediate. We use the notation from the proof of Proposition 4.1. Recall that the Jordan curve $c_{1}$ associated to the loxodromic cyclic group $\Xi_{1}=\Theta \cap \Phi_{1}$ separates $\Lambda\left(\Gamma^{o}\right)$ and satisfies $c_{1} \cap \Lambda\left(\Gamma^{o}\right)=\Lambda\left(\Xi_{1}\right)$. Since $\Lambda\left(\Xi_{1}\right)$ contains two points and since $\Lambda\left(\Gamma^{o}\right)$ is connected, the two arcs of $c_{1}-\Lambda\left(\Xi_{1}\right)$ necessarily lie in different components of $\Omega\left(\Gamma^{o}\right)$, and so $\Omega\left(\Gamma^{o}\right)$ contains two components whose closures intersect. However, as every finite index subgroup of $\Gamma$ has the same limit set as $\Gamma$, no finite index subgroup of $\Gamma$ can contain two such components, and so $\Gamma^{o}$ must have infinite index in $\Gamma$.

We can also describe this essential annulus topologically. On the 2-complex $Y$, it is a collar neighborhood on $S_{1}^{o}$ of the curve $a_{1}$. Let $N$ be the 3-manifold obtained by taking a regular neighborhood of the 2-complex $Y$, and note that $N$ has $n+1$ boundary components: $n$ are surfaces parallel to $S_{1}^{o}, \ldots, S_{n}^{o}$, and there is one other, call it $T$. The essential annulus corresponding to the curve $c_{1}$ has one boundary curve in the component of $\partial N$ parallel to $S_{1}^{o}$, and has its other boundary curve in $T$.

\section{Closing remarks}

We close with some remarks.

Remark 5.1 Suppose that $\Gamma$ is a convex co-compact Kleinian group with torsion which has connected limit set and which has the property that any pair of distinct components of $\Omega(\Gamma)$ have disjoint closures. By Selberg's Lemma [24], there exists a finite index subgroup $\Gamma^{o}$ of $\Gamma$ which is torsion free. Since $\mathrm{M}_{\Gamma^{o}}$ is acylindrical, Theorem 4.2 implies that $\Gamma^{o}$ contains a quasifuchsian subgroup which is not contained in any component subgroup, and hence so does $\Gamma$.

Remark 5.2 Note that Theorem 4.2 applies to some non-compact hyperbolic 3-manifolds with 
finitely generated fundamental group, using a standard trick. Let $\Gamma$ be a finitely generated torsionfree Kleinian group which has infinite co-volume and which contains no $\mathbf{Z} \oplus \mathbf{Z}$ subgroups. By the Core Theorem of Scott [21], there exists a compact submanifold $M$ of $\mathbf{H}^{3} / \Gamma$ whose inclusion is a homotopy equivalence. Since $\Gamma$ has infinite co-volume, the boundary of $M$ is non-empty; since $\Gamma$ contains no $\mathbf{Z} \oplus \mathbf{Z}$ subgroups, $M$ cannot contain an incompressible torus, and so by Thurston's uniformization theorem (see for example Morgan [20]), there exists a convex co-compact Kleinian group $\Phi$ uniformizing $M$. If it happens that $M$ is acylindrical, Theorem 4.2 implies that there exists a closed immersed essential surface $S$ in $M$, and hence that there exists a closed essential surface $S$ in $\mathrm{M}_{\Gamma}$. Note that this argument works in the presence of parabolics, though the fundamental group of $S$ in $\Gamma$ need not be quasifuchsian in this case.

If it happens that $\Gamma$ contains no parabolic elements, let $\Theta$ be a choice of conjugacy class in $\Gamma$ of the fundamental group of $S$. By Canary's generalization [6] of Thurston's covering theorem for hyperbolic 3-manifolds, the assumption that $\Gamma$ has infinite co-volume forces $\Theta$ to be convex co-compact, and hence quasifuchsian.

Remark 5.3 It follows from work of Thurston [29] and McMullen [19] that, if $M$ satisfies the hypotheses of Theorem 4.2, there exists a unique uniformizing convex co-compact Kleinian group $\Gamma$ for $M$ in which every component of the boundary of the convex core $\mathrm{C}_{\Gamma}$ of $\Gamma$ is totally geodesic, and hence every component of $\Omega(\Gamma)$ is a round circular disc. In this situation, it is easy to see that the immersed essential surface $S$ in $M$ given by Theorem 4.2 cannot to totally geodesic, as the quasifuchsian subgroup $\Phi$ corresponding to $S$ has non-trivial intersection with some component subgroup.

\section{References}

[1] W. Abikoff, 'The uniformization theorem', Amer. Math. Monthly 88 (1981), 574-592.

[2] L. V. Ahlfors, 'Finitely generated Kleinian groups', Amer. J. Math. 86 (1964), 413-29.

[3] L. V. Ahlfors, 'The structure of a finitely generated Kleinian group', Acta Math. 122 (1969), 1-17.

[4] J. W. Anderson, 'Intersections of analytically and geometrically finite subgroups of Kleinian groups', Transactions AMS 343 (1994), pp. 87-98.

[5] J. W. Anderson, 'On the limit set intersection theorem for finitely generated Kleinian groups', Math. Research Letters 3 (1996), 675-692.

[6] R. D. Canary, 'A covering theorem for hyperbolic 3-manifolds and its applications', Topology 35 (1996), $779-806$.

[7] D. Cooper, D. Long, and A. Reid, 'Essential closed surfaces in bounded 3-manifolds', preprint.

[8] D. B. A. Epstein and A. Marden, 'Convex hulls in hyperbolic spaces, a theorem of Sullivan, and measured pleated surfaces', in Analytical and Geometrical Aspects of Hyperbolic Spaces, Cambridge University Press, 1987, 113-253.

[9] L. Greenberg, 'Fundamental polyhedra for Kleinian groups', Annals Math. 84 (1966), 433-441. 
[10] J. Hempel, 3-manifolds, Princeton University Press, 1976.

[11] W. Jaco, Lectures on three-manifold topology, Conference board of the mathematical sciences, regional conference series in mathematics, no. 43, American mathematical society, 1980.

[12] A. Marden, 'The geometry of finitely generated Kleinian groups', Annals Math. 99 (1974), 383-462.

[13] B. Maskit, 'The canonical splitting of a Kleinian groups', IHES preprint /M/92/20.

[14] B. Maskit, 'Intersections of component subgroups of Kleinian groups', in Discontinuous groups and Riemann surfaces, ed. by L. Greenberg, Annals of Mathematics Studies 79, Princeton University Press, 1974.

[15] B. Maskit, Kleinian groups, Springer-Verlag, 1988.

[16] B. Maskit, 'On boundaries of Teichmüller spaces and on Kleinian groups II', Annals Math. 91 (1970), 607-639.

[17] B. Maskit, 'Swirls and wheels', IHES preprint /M/92/12.

[18] B. Maskit, 'A theorem on planar coverings of surfaces with applications to 3-manifolds', Annals Math. 81 (1965), 341-355.

[19] C. T. McMullen, 'Iteration on Teichmüller space', Invent. Math. 99 (1990), 425-454.

[20] J.W. Morgan, 'On Thurston's uniformization theorem for three-dimensional manifolds', in The Smith Conjecture, ed. by J. Morgan and H. Bass, Academic Press, 1984.

[21] G. P. Scott, 'Compact submanifolds of 3-manifolds', J. London Math. Soc. (2) 7 (1973), 246-250.

[22] G. P. Scott, 'Subgroups of surface groups are almost geometric', J. London Math. Soc. (2) 17 (1978), 555-565.

[23] G. P. Scott and C. T. C. Wall, 'Topological methods in group theory', in Homological Group Theory, ed. by C. T. C. Wall, London Mathematical Society Lecture Notes Series, vol. 36, Cambridge University Press, 1977.

[24] A. Selberg, 'On discontinuous groups in higher dimensional symmetric spaces', in Contributions to function theory, Tata, 1960, 147-164.

[25] J-P. Serre, Trees, Springer-Verlag, Berlin, 1980.

[26] J. Stallings, Group theory and three-dimensional manifolds, Yale monograph series, volume 4, Yale University Press, 1971.

[27] P. Susskind, 'Kleinian groups with intersecting limit sets', J. d'Analyse Math. 52 (1989), 26-38.

[28] W.P. Thurston, The geometry and topology of 3-manifolds, lecture notes, Princeton University.

[29] W. P. Thurston, 'Hyperbolic geometry and 3-manifolds', in Low Dimensional Topology, ed. by R. Brown and T. L. Thicksun, London Mathematical Society Lecture Note Series 48, Cambridge University Press, 1982. 